\title{
Reglamento de seguridad para laboratorio de logística y metrología
}

Myrna Hortencia Lezama León

mlezama@upmh.edu.mx

Rafael Ordoñez Gutiérrez

rordonez@upmh.edu.mx

Citlalli Abigail Angulo Sánchez

202110003@upmh.edu.mx

César Mateiu Quezada Mendoza

201110534@upmh.edu.mx

Universidad Politécnica Metropolitana de Hidalgo

Tolcayuca - México

\section{RESUMEN}

En este artículo se muestra el diagnóstico de Seguridad e Higiene que se realizó al Laboratorio de Logística y Metrología de la Universidad Politécnica Metropolitana de Hidalgo, apegados a la normatividad mexicana, se aplica una lista de cotejo a los encargados del laboratorio y del área de mantenimiento de la universidad, dicha lista de cotejo está basada en las Normas Oficiales Mexicanas, para así identificar los requerimientos de seguridad y finalmente elaborar el Reglamento de Seguridad del laboratorio.

Palabras clave: seguridad; laboratorio; normas oficiales; reglamento. 
Reglamento de seguridad para...

\title{
Safety regulations for the logistics and metrology laboratory
}

\begin{abstract}
This article shows the Safety and Hygiene diagnosis that was made at the Logistics and Metrology Laboratory of the Universidad Politécnica Metropolitana de Hidalgo, adhering to Mexican regulations, a checklist is applied to those in charge of the laboratory and the maintenance area of the university, said checklist is based on the Official Mexican Standards, in order to identify the safety requirements and finally develop the Laboratory Safety Regulations.
\end{abstract}

Keywords: Safety; laboratory; official standards; regulation.

Artículo recibido: 02 noviembre. 2021 Aceptado para publicación: 28 noviembre 2021 Correspondencia: mlezama@upmh.edu.mx Conflictos de Interés: Ninguna que declarar 


\section{INTRODUCCIÓN}

Dentro del sector de la educación existen diferentes metodologías para la adquisición del aprendizaje, siendo la práctica un factor muy importante para este proceso, es por ello que las instituciones de educación habilitan espacios especiales conocidos como laboratorios, los cuales cuentan con el equipo y condiciones necesarias para simular y replicar los procesos que se han abordado previamente de forma teórica, todo esto dentro de un ambiente controlado que garantice la seguridad tanto de los estudiantes como del personal.

Bajo este tenor, el laboratorio Logística y Metrología fue creado como apoyo en el cumplimiento del objetivo de la carrera de Ingeniería en Logística y Transporte de la Universidad Politécnica Metropolitana de Hidalgo (UPMH) en: "Formar profesionales que sean capaces de diagnosticar, planear, diseñar y optimizar soluciones integrales de ingeniería a las problemáticas de los sistemas logísticos y de transporte de las organizaciones." (UPMH, 2021), asimismo, este espacio busca que los estudiantes adquieran las competencias establecidas en la carrera, a través de prácticas que refuercen los conocimientos teóricos adquiridos.

El contar con laboratorios en donde se ocupan equipos que requieren de cierto cuidado tanto en el uso y manejo, así como del comportamiento para evitar la exposición de riesgos en éstas áreas de trabajo, es que se debe realizar un diagnóstico de seguridad en los laboratorios, ya que como se mencionó, dichos laboratorios por los equipos y tipo de actividades a realizar en ellos, representan condiciones diferentes a las que tiene un aula de clases, lo que implica un mayor nivel de riesgo para los estudiantes. Así mismo, es importante que dentro de las condiciones de seguridad que deben existir en el laboratorio, se regule el comportamiento dentro de éste para así garantizar el bienestar tanto de la comunidad estudiantil como del personal del laboratorio, siendo el reglamento un instrumento normativo que orienta las relaciones entre los miembros de la comunidad (Araujo y Obregón, 2020), por lo que es indispensable establecer al mismo tiempo que el diagnóstico un Reglamento de Seguridad para el Laboratorio de Logística y Metrología.

\subsection{Objetivo General}

- Realizar el diagnóstico de Seguridad e Higiene del laboratorio de Logística y Metrología de la UPMH. 


\subsubsection{Objetivos Específicos}

- Identificar la normatividad de seguridad bajo la cual se debe apegar el laboratorio.

- Hacer una evaluación del estado actual de seguridad de las instalaciones.

- Identificar requerimientos de seguridad.

- Elaborar el Reglamento de Seguridad del laboratorio de Logística y Metrología.

Cabe mencionar que, al momento de desarrollar este estudio, el laboratorio aún no estaba en funcionamiento debido a la pandemia, sin embargo, este análisis se realiza de forma previa para identificar los requerimientos y asegurar se cumplan cuando inicie su uso.

\subsection{Estado Del Arte}

La seguridad industrial comprende el conjunto de normas para garantizar la seguridad e integridad de las personas en su área de trabajo o por el desempeño de su labor (Rubio, 2012), teniendo como objetivo el prevenir accidentes y enfermedades producidas a consecuencia de su trabajo.

Es por esto de la importancia de realizar un análisis de seguridad industrial en el laboratorio de Logística y Metrología para así prevenir cualquier riesgo innherente por las actividades que se realicen en dicho laboratorio. Todo esto basado en las medidas de seguridad dadas por las autoridades. Buscando de esta forma que los alumnos obtengan experiencia suficiente no sólo con las prácticas sino también conociendo las medidas de seguridad que se deben contemplar en un ambiente real y así puedan desempeñarse en sus áreas de trabajo de manera óptima.

\section{Entre los conceptos básicos relacionados con la seguridad industrial se encuentran:}

- Prevención, que de acuerdo con Díaz (2018), es actuar de forma anticipada sobre los riesgos para suprimirlos y evitar sus consecuencias perjudiciales.

- Actos inseguros, definidos en la NOM-019-STPS-2011 como acciones del trabajador que "implican una omisión o violación a un método de trabajo seguro."

- Riesgo, definido en la norma OHSAS 18001:2007 sobre Sistemas de Gestión de la Seguridad y Salud en el Trabajo- Requisitos, como "la combinación de la probabilidad de que ocurra un suceso o exposición peligrosa y la severidad del daño o deterioro de la salud que puede causar el suceso o exposición."

Al realizar un análisis de seguridad, se debe conocer el riesgo en el entorno de trabajo, identificando el nivel de cada riesgo dependiendo de la gravedad que representan. Los riesgos que se corren dependen completamente de su naturaleza y es por eso por lo que 
deben ser priorizados para poder tratarlos de manera adecuada. Conocer cada uno de estos riesgos le ayuda al encargado del laboratorio para estar preparado en caso de que cualquier incidente se llegara a presentar, de acuerdo con Ramírez (2019), los riesgos se clasifican en:

- Eléctricos. Se originan por contacto eléctrico o exposición de cables conductores y que conllevan a: incendios, choque eléctrico, quemaduras de la piel y en circunstancias graves pueden originar la muerte de la persona.

- Físicos. Se derivan por la presencia de excesivo ruido, vibración, falta de iluminación, temperatura o humedad excesivamente altas o bajas que puedan ocasionar irritación ocular o enfermedades de origen respiratorio o auditivo

- Ergonómicos. Se presentan por la ejecución de movimientos inadecuados, así como por la adopción de posturas forzadas, asociadas con la mala disposición de equipos o uso de muebles poco adecuados, que generan trastornos músculo esqueléticos en el usuario con lesiones en espalda y extremidades.

- Locativos. Son aquellos que surgen por: falta de señalización, desorden, falta de aseo en los ambientes, pisos mojados, desnivel de pisos, mala distribución o ambientes no acordes con el uso, que originan: tropiezos, contusiones, heridas, esguinces, fracturas, e incluso alergias e infecciones por falta de aseo.

\subsubsection{Diagnóstico de seguridad e higiene}

De acuerdo con la Secretaría del Trabajo y Previsión Social (STPS, 2012), el diagnóstico de la seguridad e higiene sirve para identificar las medidas de prevención que deben ser aplicadas a las instalaciones de trabajo apegados a la normatividad vigente que en el caso de México aplican las Normas Oficiales Mexicanas (NOM).

\subsubsection{Reglamento de seguridad}

La seguridad en instalaciones abarca aspectos que aseguran condiciones correctas e idóneas para la realización de una práctica de forma segura, responsable y eficiente, evitando riesgos al momento de su realización, por lo que es necesario establecer normas de seguridad y disciplina dentro de todas las áreas que integran el Laboratorio de Logística y Metrología.

El reglamento interno de laboratorio recoge una serie de normas obligatorias de conducta que han de cumplirse por parte de los estudiantes y del encargado, por lo tanto, se trata 
de un acuerdo unilateral en el que se establece un código de conducta que dicta unas normas básicas.

\subsubsection{Normatividad}

Además de considerar los lineamientos reglamentarios establecidos dentro de la institución como el cumplimiento al reglamento estudiantil de la UPMH, también se consideran las Normas Oficiales Mexicanas (NOM) que son regulaciones técnicas de observancia obligatoria en México, aplicadas a procesos y servicios, las cuales buscan garantizar la seguridad y bienestar de las personas (Secretaría de Salud, 2015).

Las NOM aplicables a la seguridad y salud en el trabajo son 41, las cuales han sido agrupadas como Normas de: seguridad, salud, organización, específicas y de producto, para este estudio en específico sólo aplican las que se muestran en la Tabla 1.

\section{Tabla 1}

NOM's para la seguridad y salud laboral aplicadas en este estudio

\begin{tabular}{ll}
\hline \multicolumn{1}{c}{ Número } & \multicolumn{1}{c}{ Título de la norma } \\
\hline NOM-001-STPS-2008 & Edificios, locales e instalaciones \\
NOM-002-STPS-2010 & Prevención y protección contra incendios \\
NOM-004-STPS-1999 & Sistemas y dispositivos de seguridad en maquinaria \\
NOM-006-STPS-2014 & Manejo y almacenamiento de materiales \\
NOM-022-STPS-2015 & Electricidad estática \\
NOM-034-STPS-2016 & Acceso y desarrollo de actividades de trabajadores con \\
& discapacidad \\
NOM-011-STPS-2001 & Ruido \\
NOM-025-STPS-2008 & Iluminación \\
NOM-036-STPS-2018 & Factores de riesgo ergonómico. Parte 1: Manejo manual \\
de cargas \\
NOM-017-STPS-2008 & Equipo de protección personal \\
NOM-026-STPS-2008 & Colores y señales de seguridad \\
NOM-030-STPS-2009 & Servicios preventivos de seguridad y salud
\end{tabular}

Nota. Normas Oficiales Mexicanas Aplicadas a este estudio, basado en Secretaría del Trabajo y Previsión Social (STPS, 2012). 


\section{ESTRATEGIAS METODOLÓGICAS O MATERIALES Y MÉTODOS}

Este estudio tiene un enfoque cualitativo ya que implica "la recolección de datos sin medición numérica, ...con el fin de reconstruir la realidad, tal y como lo observan los actores de un sistema” (Fernández, Baptista y Hernández, 2003).

El método de investigación es un estudio de caso intrínseco, ya que se requiere analizar la seguridad del laboratorio en específico para su puesta en funcionamiento cumpliendo los requerimientos básicos de seguridad.

El diseño de la investigación es no experimental, ya que no se manipulan las variables.

Se aplicó la metodología propuesta por Velasco (2006), para realizar el diagnóstico de seguridad donde se aplican los siguientes pasos:

a) Obtener información, tanto del centro de trabajo y su actividad como de la comisión de Seguridad e Higiene del Trabajo (SHT).

b) Recorrido por las instalaciones, para el reconocimiento de las condiciones de SHT apoyándose de listas de cotejo.

c) Determinar cuáles elementos de SHT normados están presentes.

d) Contar con listados de verificación de cada una de las normas que aplican.

e) Contestar en el listado de verificación para cada uno de los requisitos.

f) Elaborar un informe que muestre el análisis de la información y la interpretación de los resultados de la revisión de campo que se hizo.

g) Elaborar el programa de SHT con los hallazgos del estudio y las recomendaciones, plasmando las acciones en un programa de acuerdo con su prioridad y con la inversión que impliquen, considerando si se requiere de expertos.

El escenario de este estudio son las instalaciones del laboratorio de Logística y Metrología, el cual ya se encuentra instalado, pero aún no ha entrado en funcionamiento debido a la pandemia por lo que se está trabajando a distancia. En cuanto a los participantes para este estudio, el instrumento será aplicado a los encargados de mantenimiento y de seguridad de la universidad, el resto del llenado del instrumento será a través de la inspección física.

Para obtener la información se elaboró una lista de cotejo basada en las NOM aplicables para el estudio de seguridad en las instalaciones, las cuales se mencionan en la Tabla 1 y a través de la observación, así como de la entrevista al personal mencionado, es que se realizó su llenado. 
Finalmente, para la elaboración del reglamento de seguridad, se realiza con la información obtenida a través de la lista de cotejo y se complementa con el Reglamento de estudiantes (UPMH, 2021).

\section{RESULTADOS Y DISCUSIÓN}

La lista de cotejo aplicada en este estudio se muestra en la tabla 2, junto con las respuestas obtenidas.

\section{Tabla 2}

Lista de cotejo para el diagnóstico

\begin{tabular}{|c|c|c|c|c|c|}
\hline Norma & Apartado & Parámetro & $\mathbf{S i}$ & No & Observaciones \\
\hline \multirow{4}{*}{$\begin{array}{l}\text { Nom- } \\
\text { 001- } \\
\text { stps- } \\
2008\end{array}$} & 7.1 & $\begin{array}{l}\text { Tanto el área de trabajo } \\
\text { como las áreas comunes se } \\
\text { encuentran totalmente } \\
\text { limpias. }\end{array}$ & $X$ & & \\
\hline & 7.2 & $\begin{array}{l}\text { Las áreas de trabajo que } \\
\text { involucran riesgo para los } \\
\text { usuarios están delimitadas } \\
\text { de manera correcta. }\end{array}$ & & $\mathrm{X}$ & $\begin{array}{c}\text { Falta señalizar } \\
\text { espacios de pasillos } \\
\text { y maquinaria }\end{array}$ \\
\hline & 7.3 & $\begin{array}{l}\text { Las instalaciones cuentan } \\
\text { con acceso y disposición } \\
\text { para personas con } \\
\text { discapacidad. }\end{array}$ & $\mathrm{X}$ & & \\
\hline & 8 & $\begin{array}{l}\text { El laboratorio cuenta con } \\
\text { sistema de ventilación } \\
\text { artificial funcionando } \\
\text { correctamente. }\end{array}$ & & $\mathrm{X}$ & $\begin{array}{l}\text { La ventilación es } \\
\text { natural, no requiere } \\
\text { ventilación } \\
\text { artificial. }\end{array}$ \\
\hline \multirow{2}{*}{$\begin{array}{l}\text { Nom- } \\
\text { 002- } \\
\text { stps- } \\
2010\end{array}$} & 6.1 & $\begin{array}{l}\text { ¿se cumplen las } \\
\text { indicaciones del patrón y/o } \\
\text { encargado? }\end{array}$ & $\mathrm{X}$ & & \\
\hline & 6.2 & $\begin{array}{l}\text { ¿se cumplen con las } \\
\text { medidas de prevención y } \\
\text { protección contra } \\
\text { incendios establecidas por } \\
\text { el patrón o encargado? }\end{array}$ & $\mathrm{X}$ & & \\
\hline
\end{tabular}




\begin{tabular}{|c|c|c|c|c|c|}
\hline Norma & Apartado & Parámetro & Si & No & Observaciones \\
\hline & 6.3 & $\begin{array}{l}\text { ¿el alumno participa en las } \\
\text { actividades de } \\
\text { capacitación } \\
\text { entrenamiento } \\
\text { proporcionadas por el } \\
\text { patrón o encargado para la } \\
\text { prevención y protección } \\
\text { contra incendios? }\end{array}$ & & $\mathrm{X}$ & $\begin{array}{l}\text { Incorporar a } \\
\text { alumnos a la } \\
\text { capacitación }\end{array}$ \\
\hline & 6.5 & $\begin{array}{l}\text { ¿cumplen con las } \\
\text { instrucciones sobre el uso } \\
\text { y cuidado del equipo de } \\
\text { protección personal } \\
\text { proporcionado por el } \\
\text { encargado a los alumnos? }\end{array}$ & $\mathrm{X}$ & & \\
\hline & 6.7 & $\begin{array}{l}\text { ¿participan en los } \\
\text { simulacros de emergencias } \\
\text { de incendio? }\end{array}$ & $\mathrm{X}$ & & \\
\hline & 6.8 & $\begin{array}{l}\text { ¿los alumnos bloquean, } \\
\text { dañan, inutilizan o dan uso } \\
\text { inadecuado a los equipos } \\
\text { de protección personal } \\
\text { para la atención a } \\
\text { emergencias, croquis, } \\
\text { planos, mapas, y } \\
\text { señalamientos de } \\
\text { evacuación, prevención y } \\
\text { combate de incendios, } \\
\text { entre otros? }\end{array}$ & & $\mathrm{X}$ & \\
\hline & 6.9 & $\begin{array}{l}\text { ¿los alumnos ponen en } \\
\text { práctica el procedimiento } \\
\text { de alertamiento, en caso de } \\
\text { detectar una situación de } \\
\text { emergencia de incendio? }\end{array}$ & $\mathrm{X}$ & & \\
\hline \multirow{3}{*}{$\begin{array}{l}\text { Nom- } \\
\text { 004- } \\
\text { stps- } \\
1999\end{array}$} & 6.1 & $\begin{array}{lr}\text { ¿los encargados } & \text { están } \\
\text { constantemente } & \text { en } \\
\text { capacitación? } & \end{array}$ & $X$ & & $\begin{array}{l}\text { Verificar se } \\
\text { integren al plan de } \\
\text { capacitación contra } \\
\text { riesgos }\end{array}$ \\
\hline & 6.2 & $\begin{array}{l}\text { ¿se cumple con las } \\
\text { medidas de seguridad e } \\
\text { higiene dentro de las } \\
\text { instalaciones? }\end{array}$ & $\mathrm{X}$ & & \\
\hline & 6.4 & $\begin{array}{l}\text { ¿se utiliza adecuadamente } \\
\text { el equipo de protección } \\
\text { personal? }\end{array}$ & $\mathrm{X}$ & & \\
\hline
\end{tabular}




\begin{tabular}{|c|c|c|c|c|c|}
\hline Norma & Apartado & Parámetro & $\mathbf{S i}$ & No & Observaciones \\
\hline & 6.5 & $\begin{array}{l}\text { ¿el personal utiliza el } \\
\text { cabello recogido? }\end{array}$ & $\mathrm{X}$ & & \\
\hline & 6.5 & $\begin{array}{l}\text { ¿los usuarios evitan el uso } \\
\text { de anillos, pulseras o } \\
\text { mangas largas, mismas } \\
\text { que representan un riesgo } \\
\text { en la operación? }\end{array}$ & $\mathrm{X}$ & & \\
\hline \multirow{4}{*}{$\begin{array}{l}\text { Nom- } \\
\text { 006-stps- } \\
2014\end{array}$} & 5.1 & $\begin{array}{l}\text { El laboratorio cuenta con } \\
\text { un programa específico } \\
\text { para dar mantenimiento a } \\
\text { los equipos que este } \\
\text { utiliza. }\end{array}$ & & $X$ & $\begin{array}{l}\text { Elaborar plan de } \\
\text { mantenimiento } \\
\text { preventivo de los } \\
\text { equipos de } \\
\text { laboratorio. }\end{array}$ \\
\hline & 5.6 & $\begin{array}{l}\text { Existe una supervisión } \\
\text { constante que asegure el } \\
\text { uso correcto de la } \\
\text { maquinaria. }\end{array}$ & $\mathrm{X}$ & & \\
\hline & 5.8 & $\begin{array}{l}\text { El laboratorio cuenta con } \\
\text { un manual de primeros } \\
\text { auxilios con base en el } \\
\text { riesgo expuesto. }\end{array}$ & & $\mathrm{X}$ & $\begin{array}{l}\text { Generar manual de } \\
\text { primeros auxilios } \\
\text { aunque se cuenta } \\
\text { con servicio médico } \\
\text { en la universidad. }\end{array}$ \\
\hline & 7.8 & $\begin{array}{l}\text { Las medidas de seguridad } \\
\text { que implementa el } \\
\text { laboratorio son adecuadas. }\end{array}$ & $\mathrm{X}$ & & \\
\hline \multirow{2}{*}{$\begin{array}{l}\text { Nom- } \\
\text { 022- } \\
\text { stps- } \\
2015\end{array}$} & 5.2 & $\begin{array}{l}\text { El laboratorio cuenta con } \\
\text { un sistema de protección } \\
\text { contra } \\
\text { eléctricas. }\end{array}$ & & $X$ & \\
\hline & 5.4 & $\begin{array}{l}\text { Se mantiene informado a } \\
\text { los alumnos sobre los } \\
\text { riesgos eléctricos que } \\
\text { existen dentro del } \\
\text { laboratorio. }\end{array}$ & $X$ & & \\
\hline $\begin{array}{l}\text { Nom- } \\
\text { 034-stps- } \\
\text { 2016, }\end{array}$ & 6.2 & $\begin{array}{l}\text { ¿se llevan a cabo acciones } \\
\text { preventivas y correctivas } \\
\text { para preservar la salud y la } \\
\text { seguridad de los usuarios? }\end{array}$ & $\mathrm{X}$ & & \\
\hline
\end{tabular}




\begin{tabular}{|c|c|c|c|c|c|}
\hline Norma & Apartado & Parámetro & $\mathbf{S i}$ & No & Observaciones \\
\hline & 6.3 & $\begin{array}{llr}\text { ¿se llevan a } & \text { cabo } \\
\text { adiestramientos y } & \text { planes } \\
\text { de atención } & \text { de } \\
\text { emergencias que } & \text { se } \\
\text { puedan suscitar en las } \\
\text { instalaciones? }\end{array}$ & $\mathrm{X}$ & & \\
\hline \multirow{3}{*}{$\begin{array}{l}\text { Nom- } \\
011- \\
\text { stps- } \\
2001\end{array}$} & 6.1 & $\begin{array}{l}\text { Todos colaboran en los } \\
\text { procedimientos } \\
\text { evaluación y observan las } \\
\text { medidas del programa de } \\
\text { conservación de la } \\
\text { audición. }\end{array}$ & & $X$ & $\begin{array}{c}\text { No hay exposición a } \\
\text { altos niveles de } \\
\text { ruido. }\end{array}$ \\
\hline & 6.2 & $\begin{array}{l}\text { Todos se someten a los } \\
\text { exámenes médicos } \\
\text { necesarios de acuerdo con } \\
\text { el programa r de } \\
\text { conservación de la } \\
\text { audición. }\end{array}$ & & $X$ & \\
\hline & 6.3 & $\begin{array}{l}\text { Todos utilizan el equipo de } \\
\text { protección personal } \\
\text { auditiva proporcionado } \\
\text { por el patrón, de acuerdo } \\
\text { con las instrucciones para } \\
\text { su uso, mantenimiento, } \\
\text { limpieza, cuidado, } \\
\text { reemplazo y limitaciones. }\end{array}$ & & $\mathrm{X}$ & \\
\hline \multirow{2}{*}{$\begin{array}{l}\text { Nom- } \\
\text { 025- } \\
\text { stps- } \\
2008\end{array}$} & 5.7 & $\begin{array}{l}\text { La iluminación dentro del } \\
\text { laboratorio es adecuada } \\
\text { para el trabajo a realizar }\end{array}$ & $\mathrm{X}$ & & \\
\hline & 5.10 & $\begin{array}{l}\text { El laboratorio cuenta con } \\
\text { un plan de mantenimiento } \\
\text { de iluminación }\end{array}$ & $\mathrm{X}$ & & $\begin{array}{c}\text { Programa de } \\
\text { mantenimiento de la } \\
\text { upmh }\end{array}$ \\
\hline \multirow[t]{2}{*}{$\begin{array}{c}\text { nom-036- } \\
\text { 1-stps- } \\
2018\end{array}$} & 6.2 & $\begin{array}{l}\text { Se da aviso de inmediato } \\
\text { al patrón y/o a la comisión } \\
\text { de seguridad e higiene } \\
\text { sobre las condiciones } \\
\text { inseguras que adviertan } \\
\text { durante el desarrollo de } \\
\text { sus actividades. }\end{array}$ & $X$ & & \\
\hline & 6.3 & $\begin{array}{l}\text { Se mantienen ordenados, } \\
\text { limpios y libres de }\end{array}$ & $X$ & & \\
\hline
\end{tabular}




\begin{tabular}{|c|c|c|c|c|c|}
\hline Norma & Apartado & Parámetro & $\mathbf{S i}$ & No & Observaciones \\
\hline & & $\begin{array}{l}\text { obstáculos sus lugares de } \\
\text { trabajo y áreas comunes }\end{array}$ & & & \\
\hline & 6.4 & $\begin{array}{l}\text { Todos desempeñan su } \\
\text { trabajo de manera segura } \\
\text { para evitar riesgos. }\end{array}$ & $\mathrm{X}$ & & \\
\hline \multirow{6}{*}{$\begin{array}{l}\text { Nom- } \\
\text { 017- } \\
\text { stps- } \\
2008\end{array}$} & 5.4 & $\begin{array}{l}\text { Dentro de las instalaciones } \\
\text { se encuentra el equipo } \\
\text { adecuado que garantice la } \\
\text { seguridad de los } \\
\text { estudiantes }\end{array}$ & $\mathrm{X}$ & & \\
\hline & 5.5 & $\begin{array}{l}\text { Se capacita y adiestra a los } \\
\text { estudiantes de manera } \\
\text { correcta acerca del uso de } \\
\text { la maquinaria dentro del } \\
\text { laboratorio. }\end{array}$ & $X$ & & \\
\hline & 5.6 & $\begin{array}{l}\text { Los responsables del } \\
\text { laboratorio dan una } \\
\text { supervisión continua a los } \\
\text { estudiantes. }\end{array}$ & $X$ & & \\
\hline & 6.1 & $\begin{array}{l}\text { ¿participan en la } \\
\text { capacitación } \\
\text { adiestramiento que el } \\
\text { patrón proporciona para el } \\
\text { uso, revisión, reposición, } \\
\text { limpieza, limitaciones, } \\
\text { mantenimiento, resguardo } \\
\text { y disposición final del } \\
\text { equipo de protección } \\
\text { personal? }\end{array}$ & & $\mathbf{X}$ & Buscar capacitación \\
\hline & 6.2 & 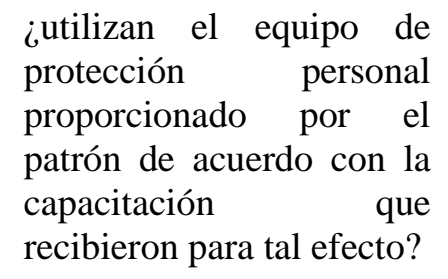 & $\mathrm{X}$ & & $\begin{array}{l}\text { Capacitación básica } \\
\text { proporcionada por } \\
\text { el encargado }\end{array}$ \\
\hline & 6.3 & $\begin{array}{l}\text { ¿revisar antes de iniciar, } \\
\text { durante y al finalizar su } \\
\text { turno de trabajo, las } \\
\text { condiciones del equipo de } \\
\text { protección personal que } \\
\text { utiliza? }\end{array}$ & $\mathrm{X}$ & & $\begin{array}{l}\text { Involucrar check list } \\
\text { al inicio del trabajo } \\
\text { ya que actualmente } \\
\text { sólo es inspección } \\
\text { visual sin registro }\end{array}$ \\
\hline
\end{tabular}




\begin{tabular}{|c|c|c|c|c|c|}
\hline Norma & Apartado & Parámetro & $\mathbf{S i}$ & No & Observaciones \\
\hline & 6.4 & $\begin{array}{l}\text { ¿informan al patrón } \\
\text { cuando las condiciones del } \\
\text { equipo de protección } \\
\text { personal ya no lo protejan, } \\
\text { a fin de que se le } \\
\text { proporcione } \\
\text { mantenimiento, o se lo } \\
\text { reemplace? }\end{array}$ & $X$ & & \\
\hline \multirow{3}{*}{$\begin{array}{l}\text { Nom- } \\
\text { 019- } \\
\text { stps- } \\
2011\end{array}$} & 6.1 & $\begin{array}{l}\text { ¿se tienen designados a } \\
\text { representantes que se } \\
\text { encarguen } \\
\text { cumplimiento de del } \\
\text { seguridad y la higiene en } \\
\text { las instalaciones? }\end{array}$ & $\mathrm{X}$ & & $\begin{array}{l}\text { Involucrar a } \\
\text { encargados y } \\
\text { alumnos }\end{array}$ \\
\hline & 6.4 & $\begin{array}{l}\text { ¿se llevan a cabo acciones } \\
\text { para la prevención } \\
\text { de problemas de la } \\
\text { seguridad y la salud? }\end{array}$ & $\mathrm{X}$ & & $\begin{array}{l}\text { El servicio médico } \\
\text { proporciona } \\
\text { capacitación }\end{array}$ \\
\hline & 6.6 & $\begin{array}{lcr}\text { ¿todos los } & \text { usuarios } \\
\text { atienden las } & \text { medidas } \\
\text { preventivas de } & \text { seguridad } \\
\text { y salud } & \text { en las } \\
\text { instalaciones? } & & \end{array}$ & $\mathrm{X}$ & & \\
\hline
\end{tabular}

\begin{tabular}{|c|c|c|c|c|c|}
\hline \multirow[t]{2}{*}{$\begin{array}{l}\text { Nom- } \\
\text { 026- } \\
\text { stps- } \\
\text { 2008, }\end{array}$} & 5.3 & $\begin{array}{l}\text { La aplicación del color, la } \\
\text { señalización y la } \\
\text { identificación de la tubería } \\
\text { están sujetos a un } \\
\text { mantenimiento que asegure } \\
\text { en todo momento su } \\
\text { visibilidad y legibilidad. }\end{array}$ & $X$ & & \\
\hline & 6.2 & $\begin{array}{l}\text { Respetar y aplicar los } \\
\text { elementos de señalización } \\
\text { establecidos por el patrón. }\end{array}$ & $X$ & & \\
\hline $\begin{array}{l}\text { Nom- } \\
\text { 030- } \\
\text { stps- } \\
2009\end{array}$ & 11.2 & $\begin{array}{l}\text { El laboratorio cuenta con } \\
\text { botiquín de primeros } \\
\text { auxilios equipado y con las } \\
\text { características } \\
\text { correspondientes. }\end{array}$ & & $X$ & Solicitar \\
\hline
\end{tabular}

Fuente: elaboración propia, basada en STPS (1999, 2003, 2008a, 2008b, 2008c, 2008d, 2009, 2010, 2011, 2012, 2014, 2015, 2016, 2018). 
De acuerdo con los resultados obtenidos en la lista de cotejo, se identifican los siguientes requerimientos a implementar de forma inmediata en el Laboratorio de Logística y Metrología:

a) Extintor, por el riesgo de incendio que tiene el laboratorio debido a la maquinaria, equipo y material de empaque se elige un extintor de $\mathrm{CO} 2$, también conocido como nieve carbónica ya que por sus propiedades es ideal para la extinción de fuegos en aparatos eléctricos de baja tensión (PRESMAN, 2019), como son las bandas transportadoras y los equipos electrónicos.

b) Botiquín de primeros auxilios, es importante contar con un botiquín de primeros auxilios dentro del laboratorio en caso de emergencia, a pesar de que la universidad cuenta con servicio médico, el botiquín queda para la atención inmediata.

c) Señalización de pasillos y espacios de trabajo, se deberán marcar los pasillos del área de almacenamiento, así como es espacio designado para la maquinaria (bandas transportadoras), para ello se elige el uso de la cinta amarilla vinílica de seguridad industrial de 2 pulgadas

d) Señalamientos de seguridad industrial y protección civil, estos letreros se colocarán dentro del área del Laboratorio de Logística y Metrología, así como en el almacén de resguardo que se encuentra dentro de dicho laboratorio, incluyen señales de información, prohibición, precaución y obligación.

Las figuras 1 y 2 muestran diferentes vistas del layout del laboratorio de Logística y Metrología.

\section{Figura 1}

\section{Laboratorio de Logística y Metrología (vista superior)}

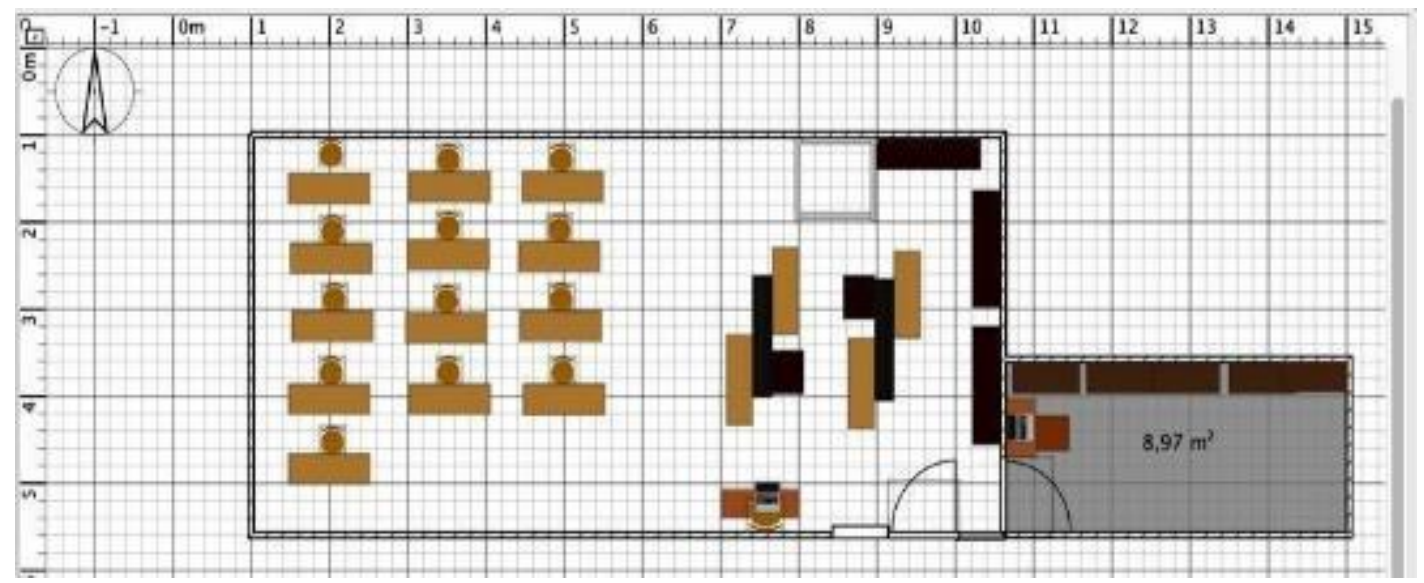




\section{Figura 2}

Laboratorio de Logística y Metrología (3D)

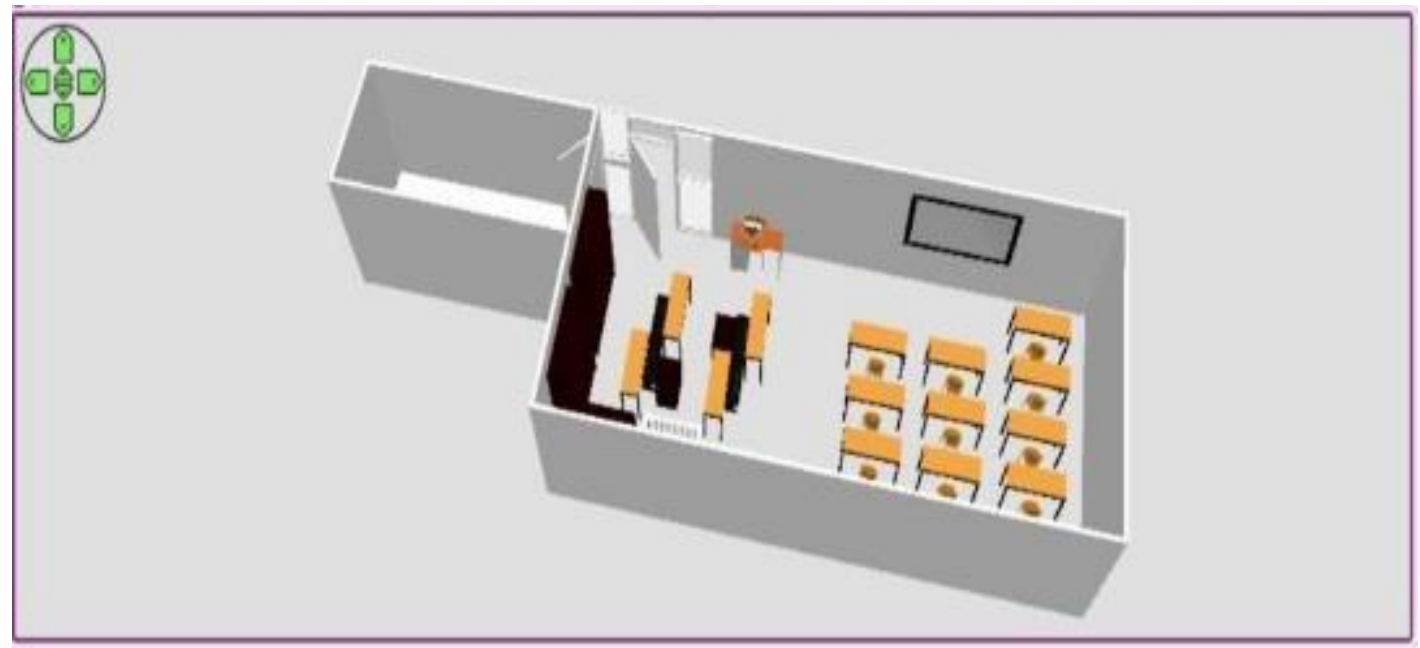

En las figuras anteriores ( 1 y 2 ), se pueden observar las mesas de trabajo y en color obscuro se representan a las bandas transportadoras, así como los racks de almacenamiento, finalmente también se observa un anexo destinado al almacén de resguardo.

Los letreros serán ubicados como se presenta en las figuras 3, 4 y 5.

\section{Figura 3}

Posición de señales pared posterior

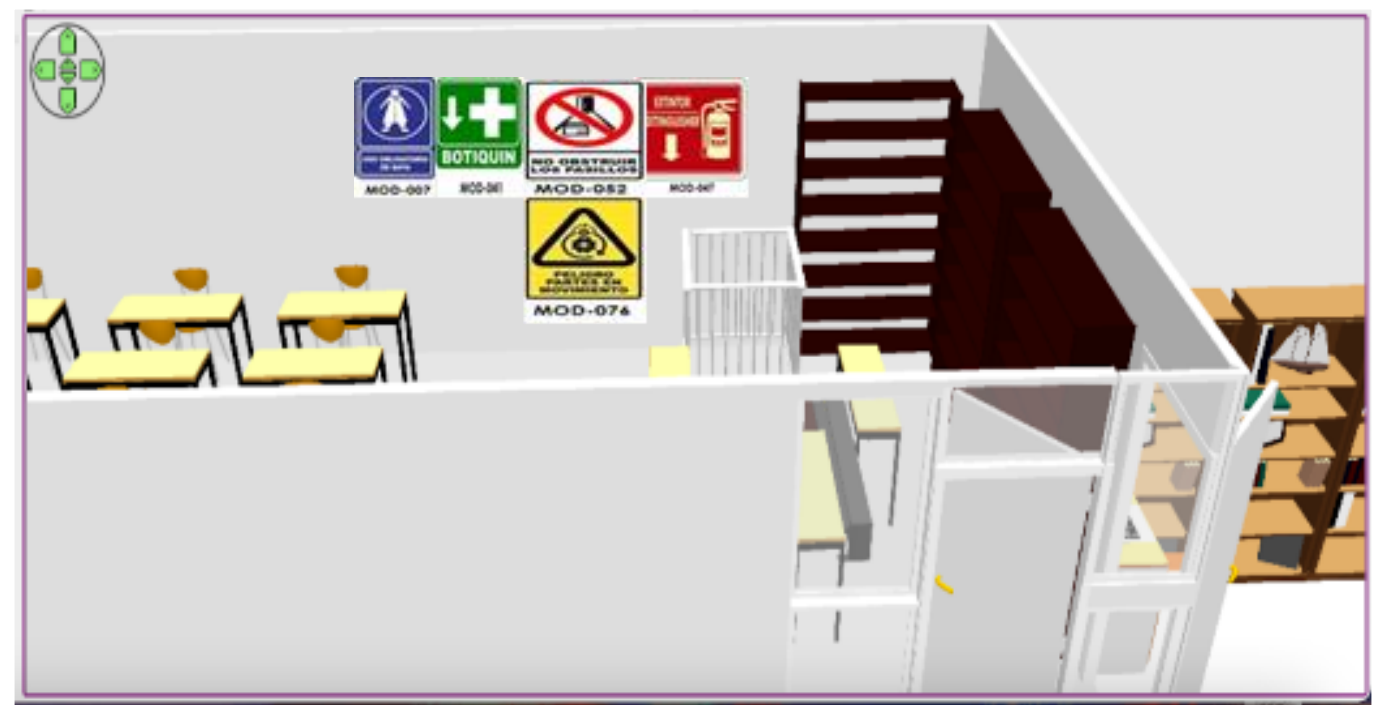




\section{Figura 4}

Posición de señales pared frontal

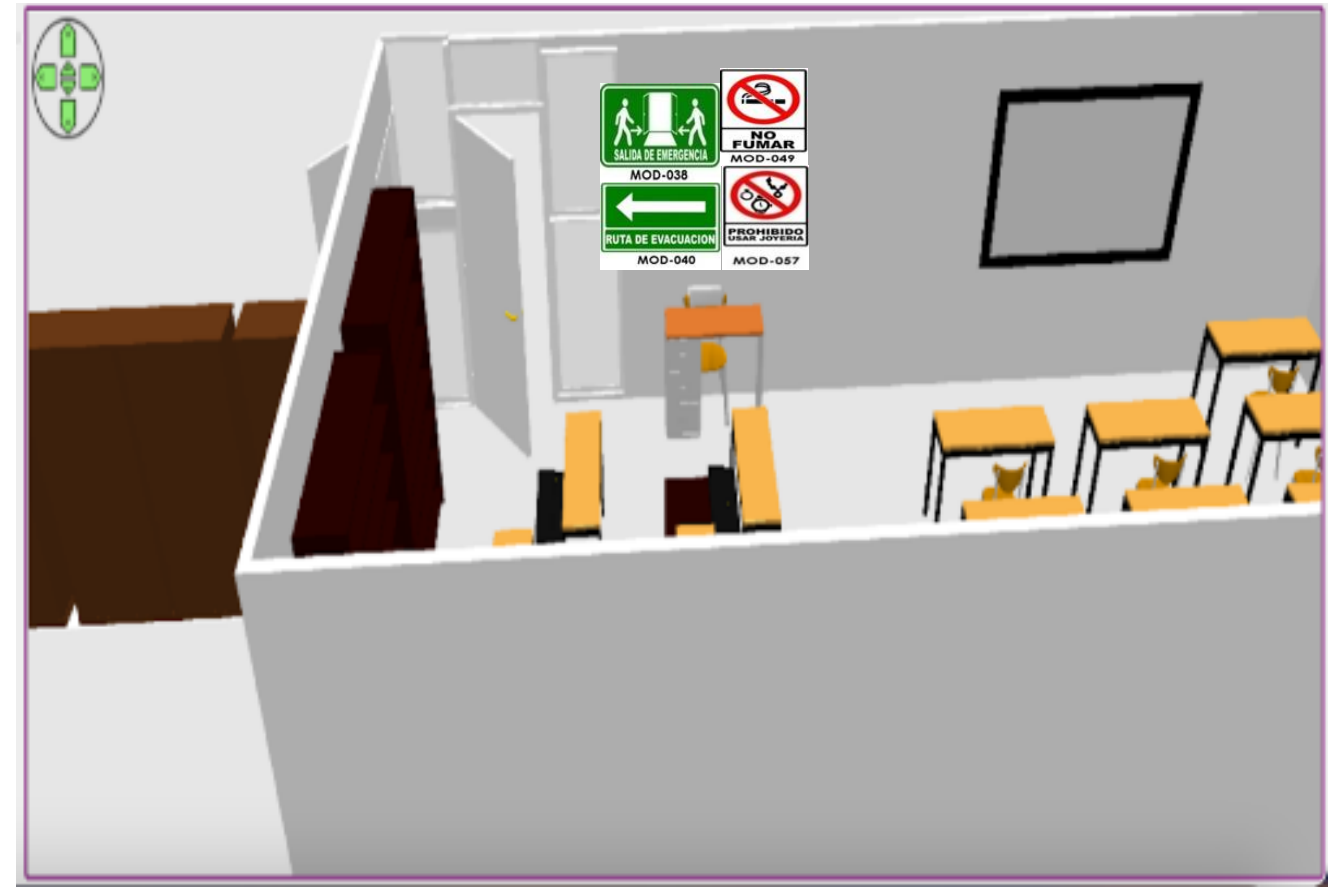

\section{Figura 5}

Letrero en Almacén de Resguardo

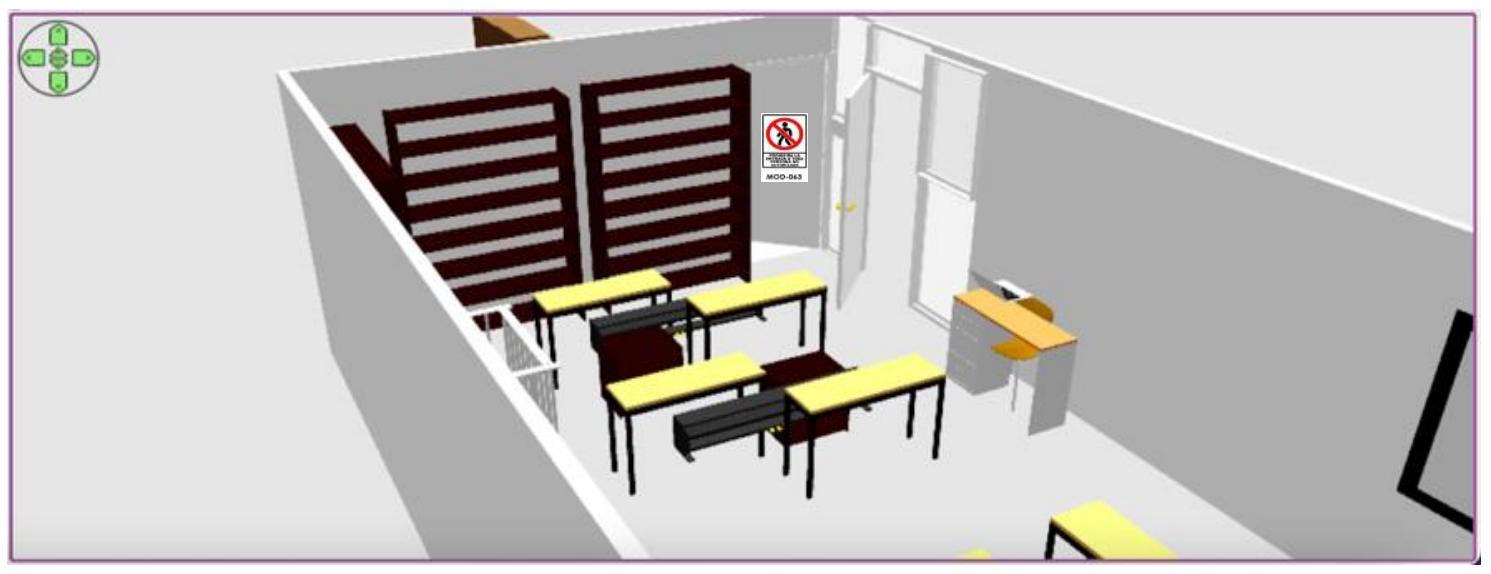

Se señalarán los pasillos del área de almacenamiento y de las bandas transportadoras, utilizando la cinta amarilla como se muestra en la figura 6. 


\section{Figura 6}

\section{Señalización de pasillos}

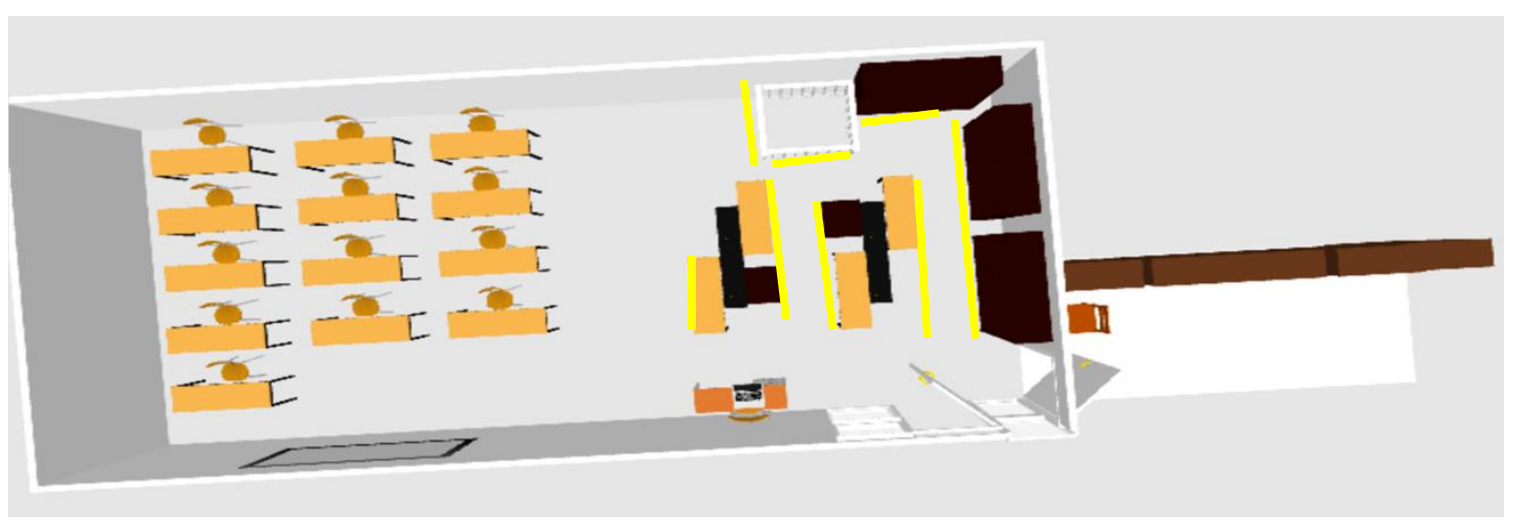

\section{Reglamento del laboratorio de Logística y Metrología}

En el presente reglamento se aborda la normatividad necesaria para la preservación de la seguridad y la salud dentro de las instalaciones del laboratorio de Logística y Metrología de la UPMH, con el fin de brindar un servicio adecuado y de calidad a sus usuarios, así como para el mayor aprovechamiento de las instalaciones.

Este reglamento es de observancia obligatoria para los usuarios del laboratorio de Logística y Metrología, además de que complementa al reglamento estudiantil de la UPMH.

\section{De los derechos.}

1. Tener acceso a las instalaciones, sin ningún tipo de discriminación.

Il. Contar con instalaciones en buenas condiciones para la realización de las distintas prácticas.

Ill. Contar con el equipo y materiales necesarios para la realización de las prácticas.

IV. Tienen derecho a recibir asesoría de los encargados sin distinción alguna.

\section{De las obligaciones}

I. Se deben cumplir con todas las medidas que señale este reglamento de seguridad e higiene, para el uso adecuado de las instalaciones.

Il. Es necesario reportar al responsable del laboratorio cualquier anomalía en el funcionamiento de los elementos que hay dentro del laboratorio.

IIl. Utilizar adecuadamente el equipo de protección personal.

IV. Evitar el uso de cabello suelto, cadenas, anillos, pulseras, mangas sueltas y otros objetos que puedan ser factor de riesgo durante las actividades. 
V. Es necesario atender las medidas preventivas de accidentes por parte de todos los usuarios.

VI. Participar en las actividades de capacitación y entrenamiento proporcionadas por el patrón para la prevención y protección contra incendios.

VII. Auxiliar en la respuesta a emergencias de incendio que se presenten en el centro de trabajo, conforme a la capacitación y entrenamiento recibidos.

VIII. Participar en los simulacros de emergencias de incendio.

IX. No bloquear, dañar, inutilizar o dar uso inadecuado a los equipos de protección personal para la atención a emergencias, croquis, planos, mapas y señalamientos de evacuación, prevención y combate de incendios, entre otros.

X. Participar en la capacitación y adiestramiento que el patrón les proporcione.

XI. Notificar al encargado de laboratorio, de conformidad con el procedimiento que para tal efecto se establezca, cualquier situación anormal que detecten en los sistemas de puesta a tierra y sistema de protección contra descargas eléctricas atmosféricas.

XII. Revisar antes de iniciar, durante y al finalizar su turno de trabajo, las condiciones del equipo de protección personal que utiliza.

\section{De las sanciones}

Las sanciones se llevarán a cabo de acuerdo con lo establecido en el Capítulo ll, Artículo 6 del Reglamento de estudiantes de la Universidad Politécnica Metropolitana de Hidalgo.

l. Amonestación verbal o escrita.

Il. Realizar actividades de apoyo y servicio social a la comunidad.

Ill. Reposición, pago o reestructuración de los bienes de la universidad dañados o perdidos.

IV. Anulación de calificaciones o créditos obtenidos fraudulentamente, así como los que se hayan obtenido en asignaturas posteriores a las anuladas.

V. Suspensión hasta por un año, de los derechos escolares.

IV. Expulsión definitiva de la universidad.

VII. Si la naturaleza de la falta constituye un delito de cualquier orden, el infractor será sancionado de acuerdo con el presente reglamento, sin menoscabo de lo que determinen las autoridades correspondientes. 


\section{CONCLUSIÓN O CONSIDERACIONES FINALES}

Al concluir el presente reglamento hemos comprendido que el Laboratorio de Logística y Metrología es un espacio destinado a la práctica y análisis de actividades que se desempeñan a lo largo de la vida laboral de los Ingenieros en Logística y Transporte, por lo anterior, es de suma importancia que los alumnos tengan un espacio de calidad y óptimas condiciones para desempeñar adecuadamente sus prácticas, sin ningún obstáculo que ponga en riesgo su seguridad e integridad personal.

El presente estudio apoyó a identificar algunas de las necesidades en seguridad para el laboratorio, las cuales serán cubiertas antes de que éste entre en función, sin embargo, hay otros requerimientos que por su naturaleza serán desarrollados posteriormente para propiciar un ambiente adecuado para los usuarios.

Entre los requerimientos que se deben tomar en cuenta para reforzar la seguridad en el laboratorio se identifican las siguientes necesidades:

- Elaborar un programa de capacitación en seguridad para todos los usuarios del laboratorio.

- Incorporar a los alumnos a la capacitación contra incendios y el uso adecuado del equipo de protección personal.

- Elaborar un plan de mantenimiento preventivo para los equipos de laboratorio.

- Generar un manual de primeros auxilios, que se dé a conocer a los alumnos, a pesar de que en las instalaciones se cuenta con servicio médico.

Todos estos elementos complementan las condiciones adecuadas para desarrollar las prácticas de manera segura, evitando correr riesgos.

\section{LISTA DE REFERENCIAS}

Araujo Lara, B. G., y Obregón Coello, J. O. (2020). Elaboración del Reglamento de Seguridad y Salud en el trabajo en un laboratorio de fabricación de cosméticos (Bachelor's thesis).

Díaz, J. M. C. (2018). Técnicas de prevención de riesgos laborales. Editorial Tebar.

Fernández Collado, C., Baptista Lucio, P., \& Hernández Sampieri, R. (2014). Metodología de la Investigación. Editorial McGraw Hill.

OHSAS 18001:2007 Sistemas de Gestión de la Seguridad y Salud en el TrabajoRequisitos. Sitio web: http://www.itlp.edu.mx/sig/Normas/OHSAS_18001.pdf 
PRESMAN. (23 de agosto de 2019). presman mantenimientos y servicios. Recuperado el 04 de septiembre de 2021, de extintor de co2: https://www.extintorespresman.es/extintor-de-co2/

UPMH. (2021). Acerca de nosotros. agosto 4, 2021, de SEPH Sitio web: http://www.upmetropolitana.edu.mx/acerca-de

Ramírez, J., E. L. (08 de AGOSTO de 2019). MEDICINA USMP. Recuperado el 03 de AGOSTO de 2021, de PROTOCOLO DE SEGURIDAD PARA LABORATORIOS:

https://medicina.usmp.edu.pe/images/servicios/SubComite_Seguridad/UI_FMH _Protocolo-de-Seguridad-Laboratorio-de-Computo-Biblioteca_v1_3.pdf

Rubio Ulloa, A. D. J. (2012). Diseño y elaboración del reglamento de seguridad en el trabajo para el centro de producción de la Unidad Académica Ciencias de la Ingeniería y Aplicadas (CIYA).

Secretaría de Salud. (2015). Normas Oficiales Mexicanas. septiembre 1, 2021, de Gobierno de México Sitio web: https://www.gob.mx/salud/en/documentos/normas-oficiales-mexicanas-9705

Secretaría del Trabajo y Previsión Social. (1999). Sistemas de protección y dispositivos de seguridad en la maquinaria y equipo que se utilice en los centros de trabajo (NOM-004-STPS-1999). http://asinom.stps.gob.mx:8145/upload/noms/Nom004.pdf

Secretaría del Trabajo y Previsión Social. (2001). Condiciones de seguridad e higiene en los centros de trabajo donde se genere ruido (NOM-011-STPS-2001). http://asinom.stps.gob.mx:8145/upload/noms/Nom-011.pdf

Secretaría del Trabajo y Previsión Social. (2008a). Edificios, locales, instalaciones y áreas en los centros de trabajo- Condiciones de seguridad (NOM-001-STPS2008). http://asinom.stps.gob.mx:8145/upload/noms/Nom-001.pdf

Secretaría del Trabajo y Previsión Social. (2008b). Equipo de protección personalSelección, uso y manejo en los centros de trabajo. (NOM-017-STPS-2008). http://asinom.stps.gob.mx:8145/upload/noms/Nom-017.pdf.pdf

Secretaría del Trabajo y Previsión Social. (2008c). Condiciones de iluminación en los centros de trabajo (NOM-025-STPS-2008). http://asinom.stps.gob.mx:8145/upload/noms/Nom-025.pdf 
Secretaría del Trabajo y Previsión Social. (2008d). Colores y señales de seguridad e higiene, e identificación de riesgos por fluidos conducidos en tuberías. (NOM026-STPS-2008). http://asinom.stps.gob.mx:8145/upload/noms/Nom-026.pdf

Secretaría del Trabajo y Previsión Social. (2009). Servicios preventivos de seguridad y salud en el trabajo- Funciones y actividades.. (NOM-030-STPS-2009). http://asinom.stps.gob.mx:8145/upload/nom/32.pdf

Secretaría del Trabajo y Previsión Social. (2010). Condiciones de seguridad-Prevención y protección contra incendios en los centros de trabajo (NOM-002-STPS-2010). http://asinom.stps.gob.mx:8145/upload/nom/33.pdf

Secretaría del Trabajo y Previsión Social. (2011). Constitución, integración, organización y funcionamiento de las comisiones de seguridad e higiene (NOM019-STPS-2011). http://asinom.stps.gob.mx:8145/upload/nom/34.pdf

Secretaría del Trabajo y Previsión Social. (2012). Marco normativo de seguridad y salud en el trabajo. septiembre 1, 2021, de Secretaría del Trabajo y Previsión Social Sitio web: http://asinom.stps.gob.mx:8145/Centro/CentroMarcoNormativo.aspx

Secretaría del Trabajo y Previsión Social. (2014). Manejo y almacenamiento de materiales-Condiciones de seguridad y salud en el trabajo (NOM-006-STPS2014). http://asinom.stps.gob.mx:8145/upload/nom/42.pdf

Secretaría del Trabajo y Previsión Social. (2015). Electricidad estática en los centros de trabajo-Condiciones de seguridad. (NOM-022-STPS-2015). http://asinom.stps.gob.mx:8145/upload/nom/46.pdf

Secretaría del Trabajo y Previsión Social. (2016). Condiciones de seguridad para el acceso y desarrollo de actividades de trabajadores con discapacidad en los centros te trabajo (NOM-034-STPS-2016). http://asinom.stps.gob.mx:8145/upload/nom/47.pdf

Secretaría del Trabajo y Previsión Social. (2018). Factores de riesgo ergonómico en el Trabajo-Identificación, análisis, prevención y control. Parte 1: Manejo manual de cargas (NOM-036-STPS-2018). https://www.dof.gob.mx/nota_detalle.php\%3Fcodigo\%3D5544579\%26fecha\%3 D23/11/2018

Velasco, A. A. (2006). Diagnóstico de seguridad e higiene del trabajo listados de verificación basados en la normatividad mexicana. e-Gnosis, 4. 